http://jmscr.igmpublication.org/home/ ISSN (e)-2347-176x ISSN (p) 2455-0450 crossref DOI: https://dx.doi.org/10.18535/jmscr/v8i7.91

\title{
Nutritional assessment using scored patient generated subjective global assessment in carcinoma cervix patients
}

\author{
Authors \\ Anjali Bhola ${ }^{1}$, Ashok K Chauhan ${ }^{2}$, Manjulata Kumawat ${ }^{3}$, Paramjeet Kaur ${ }^{3}$, \\ Abhishek Soni ${ }^{4}$ \\ ${ }^{1}$ Junior Resident, PGIMS Rohtak, Haryana \\ ${ }^{2}$ MD, Senior Professor, PGIMS Rohtak, Haryana \\ ${ }^{3} \mathrm{MD}$, consultant, PGIMS Rohtak, Haryana \\ ${ }^{4} \mathrm{MD}, \mathrm{DNB}$, consultant, PGIMS Rohtak, Haryana \\ *Corresponding Author \\ Dr Anjali Bhola \\ Junior resident, PGIMS Rohtak, Haryana, India
}

\begin{abstract}
Carcinoma cervix ranks fourth in women worldwide and second in women in India. Malnutrition negatively affects the patient's prognosis. Scored PG-SGA is validated nutritional assessment tool for cancer patients.

Purpose: Nutritional assessment of carcinoma cervix patients using PGSGA and its correlation with treatment outcome.

Methodology: 60 patients of carcinoma cervix assessed for nutritional status using Scored PatientGenerated Subjective global assessment at time of presentation, at the end of treatment and three months after completion of treatment.

Results: Before treatment, $55 \%$ patients were well nourished, $45 \%$ patients were moderately malnourished. At the end of treatment, $52 \%$ patients were moderately malnourished and $45 \%$ patients were severely malnourished. At 3rd month of follow up, $67 \%$ patients were well nourished, $28 \%$ were moderately malnourished and 5\% were severely malnourished. Mean PGSGA scores before, at end of chemoradiation and at $3 \mathrm{rd}$ month follow up were 8.08, 14.50 and 8.15 respectively ( $p$-value <0.001). Complete response was seen in $90 \%$ of PGSGA stage A and $67 \%$ of PGSGA stage B patients and progressive disease developed in 3\% of PGSGA stage A and 19\% of PGSGA stage B. patients.

Conclusion: The study has noted a higher incidence of treatment related toxicities and treatment interruptions in malnourished patients. Treatment was better tolerated in well nourished patients and tumor control was better in well nourished patients. Degree of malnutrition increased shortly after treatment. Subsequently, degree of malnutrition decreased during the first three months after treatment.

Keywords: carcinoma cervix; PGSGA; nutritional assessment; chemoradiation.
\end{abstract}

\section{Introduction}

Carcinoma of uterine cervix is the fourth most common cancer in women worldwide and second most common cancer in women in India. ${ }^{[1]}$ Malnutrition is common in cancer patients and has a negative impact on disease outcome. 
Malnutrition can be related to increases in the duration of the hospital stay, reducing the costbenefit and risk-benefit ratios of anticancer treatments and is directly or indirectly responsible for excess mortality among cancer patients. ${ }^{[2]}$

According to European Society for Clinical Nutrition and Metabolism (ESPEN) guidelines, nutritional screening should be able to predict the clinical course based on nutritional status and a patient should be benefitted from nutritional treatment. $^{[3]}$

Several screening tools are available for nutrition assessment and there is no consensus among the experts upon the best way of screening the nutritional status of cancer patients and several of these tools, including the malnutrition screening tool, the malnutrition universal screening tool and the patient generated subjective global assessment, the subjective global assessment and the nutrition risk index are validated in oncology patients. ${ }^{[4-11]}$

Being a validated nutritional assessment tool scored PG-SGA for cancer patients records and summarizes weight changes, alterations in food intake, gastrointestinal symptoms (such as nausea, vomiting and diarrhoea that have persisted for two weeks) and changes in functional capacity and physical signs of malnutrition. ${ }^{24}$ These signs are assessed by a trained doctor using skinfold measurements (loss of subcutaneous fat, muscle wasting, edema). The PG-SGA consists of two components. First, the patient-generated component that is, Boxes 1-4 (weight change, food intake, nutrition impact symptoms and activities and function) designed to be completed by the patient. Second, the professional component consists of worksheets. The five Worksheets (scoring weight loss, disease and its nutritional requirements, metabolic demand and physical examination of muscle status, fat stores and fluid status) are completed by the healthcare professional. Based on the global rating, women are classified as well nourished (PG-SGA A); moderately malnourished or suspected of being malnourished (PG-SGA B); or severely malnourished (PG-SGA C). Typical scores achieved by gynaecological cancer patients range from $0-28^{[12]}$, with higher scores reflecting greater risk of malnutrition. Scores of nine or more indicate a need for nutritional intervention options and/or improved symptom management. ${ }^{[13]}$

The present study proposes assessment of nutritional status of cervix carcinoma patients by using Scored PG-SGA and its impact on the outcome of the treatment.

\section{Material and Methods}

The study was conducted on 60 patients of cervix carcinoma, attending the Department of Radiotherapy, Pt. B. D. Sharma Post Graduate Institute of Medical Sciences, Rohtak. Inclusion criteria include previously untreated patients of histologically proven carcinoma cervix, KPS $\geq 70$, normal liver function test and renal function test. Exclusion criteria include distant metastases, prior radiation, surgery or chemotherapy for the disease, pregnant or lactating patient, any comorbidity.

\section{Methodology}

Sixty patients of carcinoma cervix were assessed for nutritional status at time of presentation, at the end of treatment and three months after completion of treatment. Patients received external beam radiotherapy 50 Gy in 25 fractions over 5 weeks with concomitant cisplatin $40 \mathrm{mg} / \mathrm{m}^{2}$ intravenous weekly for 5 weeks. However, patients received supplementary EBRT 16 Gy in 8 fractions over 1.3 weeks or intracavitatory brachytherapy one week after completion of EBRT, three fractions of $7 \mathrm{~Gy}$ each, once in a week.

The nutritional assessment of patients was performed by using Scored Patient-Generated Subjective global assessment at the time of presentation and at the end of treatment and at third month of follow up.

A numerical score was determined by using parenthetical points. These scores were applied in Global assessment of patient's nutritional status by assigning a global rating Stage A (Score $<8$ or well-nourished), Stage B (Score 8-14 or 
moderately malnourished) or Stage C (Score > 14 or severely malnourished).

\section{Statistical Analysis}

The data thus obtained was used to evaluate nutrition status of patients with cervix carcinoma using Chi- Square test and ANOVA test.

\section{Observations and Results}

Nutritional assessment by Patient Generated Subjective Global

\section{Assessment (PG-SGA)}

Before treatment, $55 \%$ patients were well nourished, $45 \%$ patients were moderately malnourished and none was severely malnourished. At the end of treatment, 3\% patients were well nourished, 52\% patients were moderately malnourished and $45 \%$ patients were severely malnourished. At third month of follow up, $67 \%$ patients were well nourished, $28 \%$ were moderately malnourished and 5\% were severely malnourished. Results are shown in table 1 .

Mean PGSGA scores before concomitant chemoradiation, at end of chemoradiation and at 3rd month follow up were 8.08, 14.50 and 8.15 respectively. PGSGA scoring was done at three different intervals, before treatment, at end of treatment and at third month of follow up and since scoring was done in same group at different intervals, repeated measure ANOVA test is applied and difference in means is found to be statistically significant with p-value $<0.001$.

Before treatment, $67 \%$ patients had decreased food intake with normal food, $1.7 \%$ had decreased food intake with intake of little solid food and $31.7 \%$ had unchanged food intake. At the end of treatment, $80 \%$ patients had decreased food intake with normal food, $15 \%$ had decreased food intake with intake of little solid food and 5\% had unchanged food intake. At third month follow up, $53.3 \%$ patients had decreased food intake with normal food, $5 \%$ had decreased food intake with intake of little solid food and $1.7 \%$ had unchanged food intake and $40 \%$ patients had increased food intake. Results are shown in table 2.
Before treatment, 58\% patients had fairly normal activities and $30 \%$ had normal activities with no limitations while $10 \%$ and $2 \%$ were bedridden for less than half of day and most of the day respectively. At the end of the treatment, 52\% patients had fairly normal activities and $13 \%$ had normal activities with no limitations while $35 \%$ were bedridden for less than half of day. At third month of follow up, $87 \%$ patients had fairly normal activities, $12 \%$ were having normal activities with no limitations and only $2 \%$ patients were bedridden for less than half of day. Results are shown in table 3 .

Before treatment, $67 \%$ patients were in mild deficit category in PGSGA physical examination (muscle status and fat stores), 23\% patients were in moderate deficit category while no patient was in severe deficit category. At the end of treatment, $21.7 \%$ patients were in mild deficit category, $71.7 \%$ were in moderate deficit and $6.7 \%$ in severe deficit category. At third month follow up, $3.3 \%$ had no deficit and $76.7 \%$ were in mild deficit and $20 \%$ in moderate deficit. Results are shown in table 4.

\section{Correlation between PGSGA stage and acute skin toxicity}

Table-5 show correlation between pre-treatment PGSGA stages with acute skin toxicity noted during $5^{\text {th }}$ week of treatment. Grade 2 skin toxicity was observed in $85 \%$ of PGSGA stage A patients and $52 \%$ of PGSGA stage B patients. Grade 3 skin toxicity was observed in $15 \%$ of PGSGA stage A patients and $48 \%$ of PGSGA stage B patients. Chi square test was applied and the difference was found statistically significant with p- value $<0.001$.

\section{Correlation between PGSGA stage and Acute mucosa toxicity}

Table-6 show correlation between pretreatment PGSGA stage with acute mucosal toxicity noted during $5^{\text {th }}$ week of treatment. Grade 1 toxicity was seen in $6.06 \%$ of PGSGA stage A patients. Grade 2 mucosal toxicity was observed in $90.09 \%$ patients of PGSGA stage A and $74.07 \%$ patients of PGSGA stage B. Grade 3 mucosal toxicity was 


\section{JMSCR Vol||08||Issue ||07||Page 558-565||July}

observed in $3.03 \%$ patients of PGSGA stage A and $25.9 \%$ patients of PGSGA stage B. Chi square test was applied and the difference was found statistically significant with p- value 0.019 .

Correlation between PGSGA and disease control

Complete response was seen in $90.09 \%$ patients of PGSGA Stage -A and $66.66 \%$ patients of PGSGA Stage- B. Chi square test was applied and the difference was found statistically non-significant with p- value 0.055 .
Correlation between PGSGA stage and completion of intended treatment and treatment interruptions

Treatment interruption of $\geq 1$ week was present in $6 \%$ patients of PGSGA stage $\mathrm{A}$ and $29.6 \%$ patients of PGSGA stage B. However, no treatment interruption was seen in $93.9 \%$ patients of PGSGA stage A and $70 \%$ patients of PGSGA stage $B$.

Table 1: PGSGA score at before treatment, end of treatment and third month follow-up.

\begin{tabular}{|c|c|c|c|c|c|c|}
\hline \multirow{2}{*}{\multicolumn{3}{|c|}{ Time interval }} & \multicolumn{3}{|c|}{ PGSGA score } & \multirow[t]{2}{*}{ Total } \\
\hline & & & \multirow{2}{*}{$\begin{array}{c}\begin{array}{c}\mathbf{0 - 8} \\
\text { (stage A) }\end{array} \\
33\end{array}$} & \multirow{2}{*}{$\begin{array}{c}\begin{array}{c}9-14 \\
\text { (stage B) }\end{array} \\
27\end{array}$} & \multirow{2}{*}{$\begin{array}{c}\begin{array}{c}>14 \\
\text { (stage C) }\end{array} \\
0\end{array}$} & \\
\hline \multirow{2}{*}{\multicolumn{2}{|c|}{ Before treatment }} & Number of patients & & & & 60 \\
\hline & & $\%$ & $55.0 \%$ & $45.0 \%$ & 0 & $100.0 \%$ \\
\hline \multirow{2}{*}{\multicolumn{2}{|c|}{ End of treatment }} & Number of patients & 2 & 31 & 27 & 60 \\
\hline & & $\%$ & $3.3 \%$ & $51.7 \%$ & $45.0 \%$ & $100.0 \%$ \\
\hline \multirow{2}{*}{\multicolumn{2}{|c|}{ At 3 month follow up }} & Number of patients & 40 & 17 & 3 & 60 \\
\hline & & $\%$ & $66.7 \%$ & $28.3 \%$ & $5.0 \%$ & $100.0 \%$ \\
\hline p value & $<0.00$ & & & & & \\
\hline
\end{tabular}

Table 2: Distribution of patients based on the amount of food intake

\begin{tabular}{|c|c|c|c|c|c|c|c|}
\hline \multirow{2}{*}{\multicolumn{2}{|c|}{ Variable }} & \multicolumn{2}{|c|}{ Pre-treatment } & \multicolumn{2}{|c|}{ End of treatment } & \multicolumn{2}{|c|}{3 month follow up } \\
\hline & & Number & Percent & Number & Percent & Number & Percent \\
\hline \multirow[t]{5}{*}{$\begin{array}{l}\text { amount of food } \\
\text { intake }\end{array}$} & $\begin{array}{l}<\text { Usual, normal food, }< \\
\text { normal amount }\end{array}$ & 40 & 66.6 & 48 & 80 & 32 & 53.3 \\
\hline & <usual, little solid food & 1 & 1.7 & 9 & 15 & 3 & 5 \\
\hline & Unchanged & 19 & 31.7 & 3 & 5 & 1 & 1.7 \\
\hline & $>$ usual & 0 & 0 & 0 & 0 & 24 & 40 \\
\hline & Total & 60 & 100.0 & 601 & 100 & 60 & 100 \\
\hline p value & \multicolumn{7}{|l|}{$<0.001$} \\
\hline
\end{tabular}

Table 3: Distribution of patients based on the activities and function

\begin{tabular}{|c|c|c|c|c|c|c|c|}
\hline \multirow{2}{*}{\multicolumn{2}{|c|}{ Variable }} & \multicolumn{2}{|c|}{ Pre-treatment } & \multicolumn{2}{|c|}{ End of treatment } & \multicolumn{2}{|c|}{3 month follow up } \\
\hline & & Number & Percent & Number & Percent & Number & Percent \\
\hline \multirow{5}{*}{$\begin{array}{l}\text { activities } \\
\text { and } \\
\text { function }\end{array}$} & fairly normal activities & 35 & 58.3 & 31 & 51.7 & 52 & 86.6 \\
\hline & in bed less than half of the day & 6 & 10.0 & 21 & 35 & 1 & 1.7 \\
\hline & most of the day in bed & 1 & 1.7 & 0 & 0 & 0 & 0 \\
\hline & normal with no limitations & 18 & 30.0 & 8 & 13.3 & 7 & 11.7 \\
\hline & Total & 60 & 100.0 & 60 & 100 & 60 & 100 \\
\hline p value & $<0.001$ & & & & & & \\
\hline
\end{tabular}

Table 4: Distribution of patients based on the physical examination

\begin{tabular}{|l|l|c|c|c|c|c|c|}
\hline \multirow{2}{*}{$\begin{array}{l}\text { Variable } \\
\text { physical } \\
\text { examination }\end{array}$} & \multicolumn{2}{|c|}{ Pre-treatment } & \multicolumn{2}{c|}{ End of treatment } & \multicolumn{2}{c|}{3 month follow up } \\
\cline { 2 - 8 } & Number deficit & 6 & 10 & 0 & 0 & 2 & 3.3 \\
\cline { 2 - 8 } & Mild deficit & 40 & 66.7 & 13 & 21.7 & 46 & 76.7 \\
\cline { 2 - 8 } & Moderate deficit & 14 & 23.3 & 43 & 71.7 & 12 & 20.0 \\
\cline { 2 - 8 } & Severe deficit & 0 & 0 & 4 & 6.7 & 0 & 0 \\
\cline { 2 - 8 } & Total & 60 & 100.0 & 60 & 100 & 60 & 100 \\
\hline \multirow{2}{*}{\begin{tabular}{l} 
p value \\
\hline
\end{tabular}}
\end{tabular}


Table 5: Correlation between PGSGA stage and Acute skin toxicity

\begin{tabular}{|l|c|c|c|}
\hline Acute skin toxicity & PGSGA Stage A (33) & $\begin{array}{c}\text { PGSGA } \\
\text { Stage B (27) }\end{array}$ & $\begin{array}{c}\text { PGSGA } \\
\text { Stage C (0) }\end{array}$ \\
\hline Grade 1 & 0 & 0 & 0 \\
\hline Grade 2 & $28(84.8 \%)$ & $14(51.8 \%)$ & 0 \\
\hline Grade 3 & $5(15.2 \%)$ & $13(48.14 \%)$ & 0 \\
\hline p value & \multicolumn{3}{|c|}{$<0.001$} \\
\hline
\end{tabular}

Table 6: Correlation between PGSGA stage and Acute mucosa toxicity

\begin{tabular}{|l|c|c|c|}
\hline $\begin{array}{l}\text { Acute mucosa } \\
\text { toxicity }\end{array}$ & $\begin{array}{c}\text { PGSGA Stage } \\
\mathbf{A}(\mathbf{3 3})\end{array}$ & $\begin{array}{c}\text { PGSGA } \\
\text { Stage B(27) }\end{array}$ & $\begin{array}{c}\text { PGSGA } \\
\text { Stage C(0) }\end{array}$ \\
\hline Grade 1 & $2(6.06 \%)$ & 0 & 0 \\
\hline Grade 2 & $30(90.9 \%)$ & $20(74.07 \%)$ & 0 \\
\hline Grade 3 & $1(3.03 \%)$ & $7(25.9 \%)$ & 0 \\
\hline p value & 0.019 & \\
\hline
\end{tabular}

\section{Discussion}

Nutrition assessment serves as the basis for malnutrition diagnosis which also includes cause, severity and type of malnutrition. ${ }^{[14]}$ The cancerspecific nutrition screening tool that seems to have the most use in research and actual patient care is the Patient-Generated Subjective Global Assessment (PG-SGA). ${ }^{\text {[24] }}$

Chantragawee et al applied the Scored PatientGenerated Subjective Global Assessment (PGSGA) questionnaire in 97 gynaecologic cancer patients to find the prevalence of malnutrition. Mean PG-SGA score was 5.2+4.7. Malnutrition (PG-SGA B and C) was found in 52 patients $(53.6 \%, 95 \%$ CI $43.7 \%-63.2 \%){ }^{[15]}$

Rodrigues et al assessed nutritional status and its relationship to outcome and 1 year mortality in 146 women diagnosed with gynaecological cancers.62.4\% of the women were classified as being at nutritional risk or having moderate or severe malnutrition. The median hospital stay was statistically lower in well nourished patients. Individuals with a score above the cut-off point of 10 were 30.7 times more likely (CI 95\%:11.879.4 ) to die. There was a $52.1 \%$ rate of mortality within one year. Patients classed as having some degree of malnutrition had a significantly lower median survival. $^{[16]}$

Vigano et al found that cancer patients with higher PG-SGA scores ( $\geq 9 v s$. 0 to 1 ) had $12 \%$ greater length of hospital stay, a reduction in the doses of chemotherapy, and increased mortality. ${ }^{[17]}$
Bauer et al used the scored PG-SGA as a nutrition assessment tool in 71 patients with cancer. 17 patients were well nourished, 42 patients were moderately or suspected of being malnourished and 12 patients were severely malnourished consistent with SGA with a sensitivity of $98 \%$ and a specificity of $82 \%$ at predicting SGA classification. The median length of stay of wellnourished patients (SGA-A) was significantly lower than that of the malnourished $(\mathrm{SGA} B+\mathrm{C})$ patients $(\mathrm{p}=0.024) .{ }^{[6]}$

Das et al conducted a study in Gujarat cancer research institute, Ahmadabad, Gujarat, in 60 gynaecological cancer patients for assessment of their nutritional status and $83.3 \%$ of gynaecologic cancer cases had some degree of malnutrition or were at risk of malnutrition according to scored PG-SGA. ${ }^{[18]}$

Isenring et al studied the scored Patient-generated Subjective Global Assessment (PG-SGA) and its association with quality of life in radiotherapy receiving patients. PG-SGA score and global QoL were correlated $(\mathrm{p}<0.001)$ at baseline. There was a decrease in nutritional status according to PGSGA score $(p<0.001)$ and a decrease in global QoL ( $p<0.001$ ) after 4 weeks of radiotherapy. There was a linear trend for change in PG-SGA score $(\mathrm{p}<0.001)$ and change in global QoL ( $p$ $=0.003){ }^{[19]}$

Laky et al assessed malnutrition among gynaecological cancer patients using Scored patient-generated subjective global assessment (PG-SGA) and serum albumin before treatment. 
$116(80 \%)$ patients were categorized as PG-SGA class A, 29 (20\%) patients were PG-SGA B and none of the patients were PG-SGA C. ${ }^{[20]}$

Khoshnevis et al assessed nutritional status in cancer patients. The PG-SGA standard questionnaire was applied to 416 cancer patients. The prevalence of malnutrition among the patients was $53.1 \%$ with $29.1 \%$ being moderately and $24 \%$ being severely malnourished. $35 \%$ of the patients had over 5\% weight loss within the last mouth. The average PG-SGA score was 10.1 and 49 was the maximum score. 46.1 percent of the patients scored over 9 (requiring critical nutrient intervention). ${ }^{[21]}$

Nho et al studied the impact of malnutrition on survival in patients with gynaecologic cancer undergoing chemotherapy. PG-SGA scores were significantly lower within the survivors as compared to non-survivors $(\mathrm{p}=0.014)$. The mean PG-SGA for survivors was $10.2 \pm 5.0$ and nonsurvivors was $13.2 \pm 4.1$. $^{\text {[2] }}$

Sharma et al evaluated nutritional status of cancer patients during treatment by scored PatientGenerated Subjective Global Assessment (PGSGA). $15.8 \%$ (9) were well nourished, $31.6 \%$ (18) were moderately or suspected of being malnourished and $52.6 \%$ (30) were severely malnourished as classified by PGSGA. ${ }^{[23]}$

In our study, before treatment, 55\% patients were well nourished or PGSGA stage A; $45 \%$ patients had moderate malnutrition or PGSGA stage B; none of the patient was severely malnourished or PGSGA stage C. Mean PGSGA score was $8.08 \pm$ 2.90. At the end of treatment, $3 \%$ patients were well nourished or stage A, 52\% patients were moderately malnourished or stage B and $45 \%$ patients were severely malnourished or stage $\mathrm{C}$ according to PGSGA and mean PGSGA score at the end of treatment was $14.5 \pm 3.23$. It has also been observed that $95 \%$ patients had decreased food intake and 5\% patients had unchanged food intake over the preceding month. In our study, the most common cause of food intake reduction during the past two weeks were; decreased appetite, nausea, vomiting, diarrhea and pain abdomen. It has also been evidenced by PGSGA global assessment that only $13 \%$ patients had normal activities with no limitations at the end of treatment, $52 \%$ patients rated their activities as not their normal self but able to do fairly normal activities, 35\% patients rated their activities as not feeling up to most things, but in bed or chair less than half the day and none of the patients was pretty much bedridden and rarely out of the bed. Physical examination conducted for subjective evaluation of fat, muscle and fluid status showed that $22 \%$ patients had mild deficit, $71 \%$ patients had moderate deficit and $7 \%$ patients had severe deficit at the end of treatment. Sharma et al found similar results in cancer patients during treatment assessed by PGSGA. ${ }^{23}$ At third month follow up, $67 \%$ patients were well nourished or stage A, $28 \%$ patients were moderately malnourished or stage B and $5 \%$ patients were severely malnourished or stage C. Mean PGSGA score was 8.15 \pm 3.57 .

\section{Correlation between pretreatment PGSGA stage and acute toxicities}

Grade 3 skin toxicity was seen in $15 \%$ patients of PGSGA stage A (well nourished) and $48 \%$ patients of PGSGA stage B (moderately malnourished). Grade 3 mucosal toxicity was seen in $3 \%$ patients of PGSGA stage $\mathrm{A}$ and $26 \%$ patients of PGSGA stage B. There was more likelihood of higher radiation induced acute toxicity and resulting treatment interruption in patients who were malnourished prior to therapy. A study by Rodrigues et al identified PGSGA score as negative prognostic factor for the increased risk of toxicity from oncological treatment in women undergoing chemotherapy for gynecological cancer. ${ }^{[16]}$

\section{Correlation between pretreatment PGSGA stage and disease control}

Complete response was seen in $90 \%$ of PGSGA stage A patients and $67 \%$ of PGSGA stage B patients. Progressive disease was seen in $3 \%$ of PGSGA stage A patients and $18.5 \%$ of PGSGA stage B patients. Progressive disease developed in more of malnourished patients than well nourished patients. Nho et al also reported that nourished 
status was associated with improved survival compared to malnourished status. ${ }^{\text {[2] }}$

\section{Correlation between PGSGA stage and completion of intended treatment and treatment interruptions}

All the patients completed the intended treatment. Treatment interruption of $\geq 1$ week was present in $6 \%$ patients of PGSGA stage $\mathrm{A}$ and $29.6 \%$ patients of PGSGA stage B. However, no treatment interruption was seen in $93.9 \%$ patients of PGSGA stage A and $70 \%$ patients of PGSGA stage $B$.

\section{Conclusion}

Malnutrition was prevalent in $45 \%$ carcinoma cervix patients according to PGSGA before treatment and degree of malnutrition increased during treatment. At the end of treatment, 97\% patients were malnourished (moderate or severe malnutrition) according to PGSGA. The study has noted a statistical significant higher incidence of treatment related toxicities and treatment interruptions in malnourished patients. Treatment was better tolerated in well nourished patients and tumour control was better in well nourished patients (statistically non-significant). Complete response was seen in $90 \%$ of PGSGA stage A patients and $67 \%$ of PGSGA stage B patients and progressive disease developed in 3\% of PGSGA stage A patients and 19\% of PGSGA stage B patients. Six percent patients of PGSGA stage A had interruptions in treatment and $30 \%$ of PGSGA stage B patients had treatment interruptions.

\section{References}

1. Bray F, Ferlay J, Soerjomataram I, Siegel RL, Torre LA, Jemal A. Global cancer statistics 2018: GLOBOCAN estimates of incidence and mortality worldwide for 36 cancers in 185 countries. Cancer J Clin 2018;68: 394424.

2. Chao PC, Chuang HJ, Tsao LY, Chen PY, Hsu CF, Lin HC, et al. The Malnutrition Universal Screening Tool (MUST) and a nutrition education program for high risk cancer patients: strategies to improve dietry intake in cancer patients. Biomedicine 2015;5:30-5.

3. Kondrup J, Allison SP, Elia M, Vellas B, Planth M. ESPEN Guidelines for nutrition screening 2002. Clin Nutr 2003;415-21.

4. Amaral TF, Antunes A, Cabral S, Alves P, Kent-Smith L. An evaluation of three nutritional screening tools in a Portuguese oncology centre. J Hum Nutr Diet 2008;21:575-83

5. Bauer J, Capra S. Comparison of a malnutrition screening tool with subjective global assessment in hospitalised patients with cancer-sensitivity and specificity. Asia Pac J Clin Nutr 2003;12:257-60.

6. Bauer J, Capra S, Ferguson M. Use of the scored patient-generated subjective global assessment tool in patients with cancer. Eur J Clin Nutr 2002;56:779-85.

7. Segura A, Pardo J, Jara C, Zugazabeitia L, Carulla J, De Las Penas R, et al. An epidemiological evaluation of the prevalence of malnutrition in Spanish patients with locally advanced or metastatic cancer. Clin Nutr 2005;24:801-14.

8. Unsal D, Mentes B, Akmansu M, Uner A, Oguz M, Pak Y. Evaluation of nutritional status in cancer patients receiving radiotherapy: a prospective study. Am J Clin Oncol 2006;29:183-88

9. Sungurtekin H, Sungurtekin U, Balci C, ZencirM, Erderm E. The influence of nutritional status on complications after major intra-abdominal surgery. J Am Coll Nutr 2004;23:227-32.

10. Kyle UG, Kossovsky MP, Karsegard VL, Pichard C. Comparison of tools for nutritional assessment and screening at hospital admission: a population study. Clin Nutr 2006;25:409-17.

11. Roulston F, McDermott R. Comparison of three validated nutritional screening tools in the oncology setting. Proc Soc Nutr 2008;67(OCE7):E26. 
12. Laky B, Janda M, Cleghorn G, Obermair A. Comparison of different nutritional assessments and body composition measurements to detect malnutrition among gynaecological cancer patients. Am J Clin Nutr 2008;87:1678-85.

13. Ottery FD: Patient-Generated Subjective Global Assessment. In The Clinical Guide to Oncology Nutrition Edited by: McCallum PD, Polisena CG. Chicago: The American Dietetic Association; 2000:11-23.

14. Cederholm T, Barazzoni R, Austin P, Ballmer P, Biolo G, Bschoff SC, et al. ESPEN guidelines on definitions and terminology of clinical nutrition. Clin Nutr 2017; 36:49-64.

15. Chantragawee C, Achariyapota V. Utilization of a Scored Patient-Generated Subjective Global Assessment in Detecting a Malnourished Status in Gynecologic Cancer Patient. Asian Pac J Cancer Prev 2016; 17:4401-4

16. Rodrigues CS, Chaves GV. Patient-generated Subjective Global Assessment in relation to site, stage of the illness, reason for hospital admission, and mortality in patients with gynecological tumors. Support Care Cancer 2015;23:871-9.

17. Vigano AL, Di Tomasso J, Kilgour RD, Trutschnigg B, Lucar E, Morais JA, et al. The abridged patient-generated subjective global assessment is a useful tool for early detection and characterization of cancer cachexia. J Acad Nutr Diet 2014;114:1088-98.

18. Das U, Patel S, Dave K, Bhansali R. Assessment of nutritional status of gynecologic cancer cases in India and comparison of subjective and objective nutrition assessment parameters. South Asian J Cancer 2014;3:38-42.

19. Isenring E, Bauer J, Capra S. The scored patient-generated subjective global assessment (PG-SGA) and its association with quality of life in ambulatory patients receiving radiotherapy. Eur J Clin Nutr 2003;57:305-9.

20. Laky B, Janda M, Bauer J, Vavra C, Cleghorn G, Obermair A. Malnutrition among gynicological cancer patients. Eur Journal of Clinical Nut 2007;61:642-6.

21. Khoshnevis N, Ahmadizar F, Alizadeh M, Akbari ME. Nutritional assessment of cancer patients in Tehran, Iran. Asian Pac J Cancer Prev 2012;13:1621-6.

22. Nho JH, Kim SR, Kang GS, Kwon YS. Relationships among malnutrition, depression and quality of life in patients with gynecologic cancer receiving chemotherapy. Korean J Women Health Nurs 2014; 20:11725.

23. Sharma D, Kannan R, Tapkire R, Nath S. Evaluation of Nutritional Status of Cancer Patients during Treatment by PatientGenerated Subjective Global Assessment: a Hospital-Based Study. Asian Pac J Cancer Prev 2015;16: 8173-8176.

24. Ottery FD. Definition of standardized nutritional assessment and interventional pathways in oncology. Nutrition 1996; 12:S15-9. 